\title{
CLASIFICACIÓN DE SEGMENTOS NORMALES, HYPERKINÉTICOS E HYPOKINÉTICOS EN IMÁGENES VENTRICULOGRÁFICAS, APLICANDO EL MÉTODO DE LÍNEA CENTRAL
}

\author{
CLASSIFICATION OF HYPERKINETIC, HYPOKINETIC AND \\ NORMAL SEGMENTS IN VENTRICULOGRAPHIC IMAGES, USING \\ CENTERLINE METHOD
}

\author{
Hernando José Velandia-Villamizar \\ M. Sc. Ingeniería Biomédica \\ Universidad de Pamplona \\ Pamplona, Colombia \\ hernandovelandia@unipamplona.edu.co
}

\author{
Rubén de Jesús Medina-Molina \\ Ph. D. Procesamiento de Señales y Comunicaciones \\ Universidad de los Andes \\ Mérida, Venezuela \\ rmedina@ula.ve
}

\author{
Luis Enrique Mendoza \\ M. Sc. Ingeniería Biomédica \\ Universidad de Pamplona \\ Pamplona, Colombia \\ luis.mendoza@unipamplona.edu.co
}

Resumen- La valoración del movimiento regional de la pared ventricular izquierda es de vital importancia a nivel clínico, ya que esta cavidad es la más susceptible a sufrir daño severo en enfermedades, tales como: la hipertensión arterial, la diabetes mellitus o la aterosclerosis. Con base en este contexto, se ha desarrollado un sistema semiautomático asistido por computadora, que permite cuantificar dicho movimiento en imágenes ventriculográficas. El trabajo se focaliza en el estudio de la diástole y la sístole final. En primer lugar es realizado un proceso de segmentación manual por parte del especialista; seguidamente se cuantifican 100 radios generados entre los dos contornos ventriculares, aplicando el método de línea central, que posibilita la clasificación de estos en segmentos normales, hyperkinéticos e hypokinéticos. Finalmente, el proceso de prueba se realizó utilizando datos reales adquiridos en el Instituto Autónomo Hospital Universitario de la Universidad de los Andes IAHULA.

Palabras clave- Pared ventricular izquierda; sistema semiautomático; imágenes ventriculográficas; hyperkinésis; hypokinésis.

Abstract- Evaluation of regional left ventricular wall motion is of vital importance at the clinical level, since this cavity is the most susceptible to severe dama- ge in diseases such as arterial hypertension, diabetes mellitus and atherosclerosis. This paper reports on the design of a software platform for the estimation of parameters describing the left ventricular wall motion in ventriculographic images. The system focuses on end-diastolic and end-systolic contours. Firstly is carried out a process of manual segmentation by the specialist, then 100 chords generated between two ventricular contours are quantified, which allows the classification of these segments (normal, hyperkinetic and hypokinetic). At last, the testing process is performed using actual data acquired in the Institute Autonomy Hospital Universitary of University of the Andes IAHULA.

Keywords- left ventricular wall, software platform, ventriculographic images, hypokinesis, hyperkinesis.

\section{INTRODUCCIÓN}

La valoración morfológica y funcional del corazón es de vital importancia, debido a que el daño cardiovascular es una de las principales causas de mortalidad a nivel mundial [1]. De las cuatro cámaras cardiacas, el ventrículo izquierdo es la más susceptible a sufrir daño severo, en enfer- 
medades, tales como: la hipertensión arterial, la diabetes mellitus o la arterosclerosis [2], [3]. En la práctica clínica convencional, el movimiento de la pared ventricular izquierda es evaluado subjetivamente a partir de ventriculogramas obtenidos con rayos X [4]. La pobre reproducibilidad de tal análisis cuantitativo, ha conllevado al desarrollo de métodos que miden la magnitud del movimiento de la pared, a partir de 4 a 100 puntos alrededor del endocárdico. Uno de ellos, es el alcanzado a través de la búsqueda de una línea central o eje [5], determinado entre los contornos de las imágenes en diástole y sístole final; con base en este, es posible generar radios o segmentos que permitan medir su diferencia. Estas distancias son un estimado del movimiento de la pared ventricular [6] y permiten clasificar una región de la pared como normal, hyperkinética e hypokinética (términos referidos al aumento o reducción generalizada del grado de contracción sistólica) con relación a estudios previos realizados con pacientes que presentan una funcionalidad ventricular normal [7].

\section{DESCRIPCIÓN DEL SISTEMA DE ADQUISICIÓN DE IMÁGENES}

La ventriculografía por rayos $X$ es una modalidad ampliamente utilizada en la evaluación de la función ventricular izquierda. Para su visualización, el paciente es sometido a un cateterismo cardiaco, donde la región por estudiar es llenada con un agente de contraste radio-opaco [8]. La adquisición de las imágenes se realiza en dos planos (la vista oblicua anterior derecha (OAD) $30^{\circ}$ y la vista oblicua anterior izquierda (OAI) $\left.60^{\circ}\right)$. El tiempo de adquisición promedio es de 8 a 10 s cubriendo de 7 a 9 ciclos cardiacos. En uno de ellos se seleccionan las imágenes diástole y sístole final, con el objeto de que el especialista trace el contorno endocárdico respectivo [9]. El equipo utilizado para la adquisición y visualización del estudio de ventriculografía es el INNOVA 2000, construido por la General Electric y que presenta las siguientes características: Posee un detector digital de estado sólido Revolution, las imágenes digitales presentan un campo de vista de $20,5 \mathrm{~cm}$ x 20,5 $\mathrm{cm}$, las secuencias de imágenes se registran a 30 cuadros por segundo con una resolución de 512 x 512 pixeles y cada píxel se representa con 256 niveles de gris [10], [11].

\section{METODOLOGÍA}

El procedimiento consiste en valorar el movimiento de la pared ventricular izquierda, a través de la cuantificación de un conjunto de segmentos, generados entre los contornos diastólico y sistólico final [12].

\subsection{Selección de las imágenes angiográficas}

Del estudio de angiografía se eligen 2 imágenes: diástole y sístole final, Fig. 1(a) y 1(b), donde el especialista traza sus contornos, considerando una herramienta interactiva que permite seleccionar puntos al utilizar una interfaz gráfica.

Fig. 1. (A) IMAGEN DIASTÓLICA FINAL. (B) IMAGEN SISTÓLICA FINAL

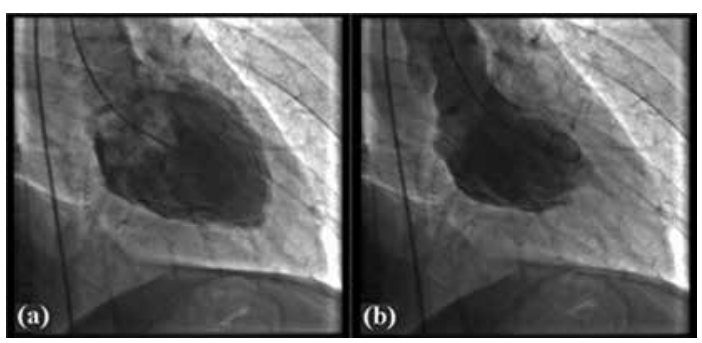

Fuente: IAHULA, Equipo Innova 2000.

\subsubsection{Calibración}

La resolución espacial (referencia) es definida como el tamaño de un píxel en el mundo real ( $\mathrm{mm}$, $\mathrm{cm}$, etc.). Esta conversión es obtenida a través de otra imagen proporcionada por el estudio de angiografía, Fig. 2(a); en ella la referencia se obtiene colocando una esfera de $4 \mathrm{~cm}$ (o de un $1 \mathrm{~cm}$ ) de diámetro al realizar el cateterismo en la región axilar izquierda del paciente. Al medir ese diámetro en pixeles es posible determinar el factor de escala requerido para la evaluación cuantitativa. Este procedimiento es realizado con base en la Transformada de Hough (TH) ([13], [14]), de la siguiente manera:

- Preprocesamiento: En primer lugar la imagen original, Fig. 2(a) es binarizada usando el algoritmo de Otsu [15], Fig. 2(b); seguidamente se realiza la extracción de una subimagen de la región de interés, Fig. 2(c) con base en la demarcación de un punto por parte del especialista, lo más cercano al centro de la esfera en la Fig. 2(a). Finalmente, a esta subimagen le es aplicado el algoritmo de Canny [16], con 
la finalidad de obtener una representación que solo contenga los puntos del contorno, Fig. 2(d).

- Aplicación de la TH: Cada uno de los puntos del contorno en la Fig. 2(d) es empleado como centro de una circunferencia, Fig. 2(e) de radio $r$ (dato de entrada proporcionado por el usuario). Todos los puntos que conforman cada una de las circunferencias generadas, son almacenados en un arreglo bidimensional (matriz de votos) del tamaño de la imagen de la Fig. 2(e). Seguidamente se procede a calcular el máximo valor de repetitividad en dicha matriz y se extrae su posición $(a, b)$ correspondiente al centro de la circunferencia. Finalmente, se genera dicho contorno con estos parámetros, Fig. 2(f) y se realiza el despliegue de estos puntos en la imagen original, Fig. 2(g).

Fig. 2. (A) IMAGEN DE REFERENCIA. (B) APLICACIÓN DEL MÉTODO DE OTSU Y SELECCIÓN DE LA SUBIMAGEN DE INTERÉS. (C) SUBIMAGEN EXTRAÍDA. (D) EMPLEO DEL MÉTODO DE CANNY. (E) APLICACIÓN DE LA TRANSFORMADA DE HOUGH. (F) DETECCIÓN DEL CONTORNO DE LA ESFERA A TRAVÉS DEL PROCEDIMIENTO REALIZADO EN (E). (G) MAPEO DEL CONTORNO OBTENIDO DE LA IMAGEN ORIGINAL

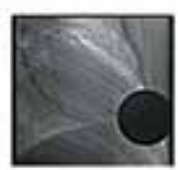

a)

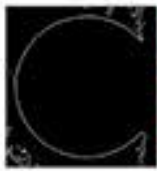

d)

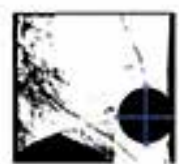

b)

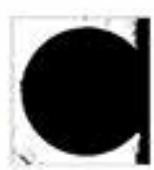

c)

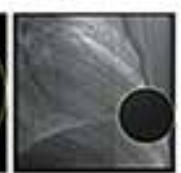

g)
Fuente: autores.

\subsection{Procesamiento de imágenes binarias}

Las figuras $3(a)$ y $3(b)$ ilustran las imágenes correspondientes al contorno diastólico y sistólico final, obtenidas por demarcación manual del especialista. Seguidamente se realiza la superposición de contornos, Fig. 3(c), con la finalidad de calcular la curva de sinergia ventricular, a través del método de línea central [17]; el procedimiento desarrollado en este trabajo para tal fin es el siguiente: las figuras $3(a)$ y $3(b)$ son convertidas a las representaciones mostradas en las Fig. 3(d) y 3(e), por medio de un algoritmo de llenado [9].
Seguidamente es aplicada la función lógica OR exclusiva a estas máscaras, obteniendo así la región de intersección entre ellas, Fig. 3(f).

Fig. 3. (A) CONTORNO DIASTÓLICO FINAL. (B) CONTORNO SISTÓLICO FINAL. (C) CONTORNOS SUPERPUESTOS. (D) CONTORNO DIASTÓLICO RELLENO. (E) CONTORNO SISTÓLICO RELLENO. (F) APLICACIÓN OR EXCLUSIVA A LAS FIGURAS (D) Y (E)

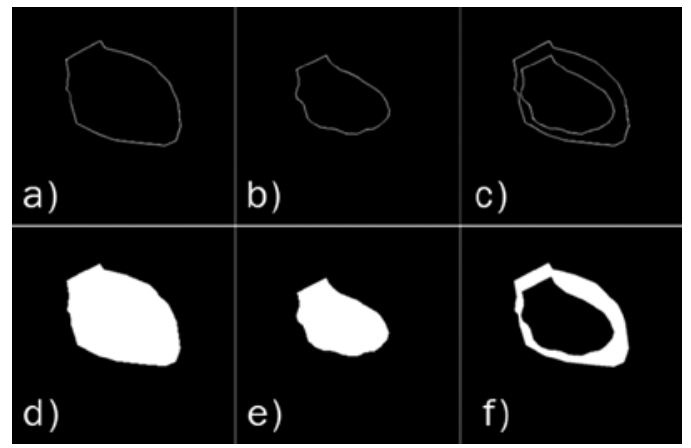

Fuente: autores.

\subsubsection{Curva de sinergia ventricular}

Para generar esta representación, se valora el ventrículo de manera regional, demarcando 100 radios entre el contorno diastólico y sistólico final, con base en la línea central o eje [5], [6], [17], de la siguiente manera: En primer lugar se divide el espacio (superposición de los contornos) en 100 puntos equidistantes, trazando radios desde el centroide diastólico hacia las coordenadas de este contorno, con una diferencia angular entre ellos de 3,6 $6^{\circ}\left(360^{\circ} / 100\right.$ (radios)), Fig. 4(a)).

Fig. 4. (A) TRAZO DE 100 RADIOS ANGULARMENTE ESPACIADOS EN EL CONTORNO DIASTÓLICO, DESDE SU CENTROIDE. (B) APLICACIÓN OR EXCLUSIVA ENTRE LAS IMÁGENES DE LAS FIGURAS 3(D) Y 3(E). (C) LÍNEA CENTRAL GENERADA. (D) SUPERPOSICIÓN DE LOS CONTORNOS Y RESULTADO FINAL DEL MÉTODO DE LÍNEA CENTRAL; ADEMÁS SE ILUSTRAN SUS DIFERENTES ZONAS DIVISORIAS

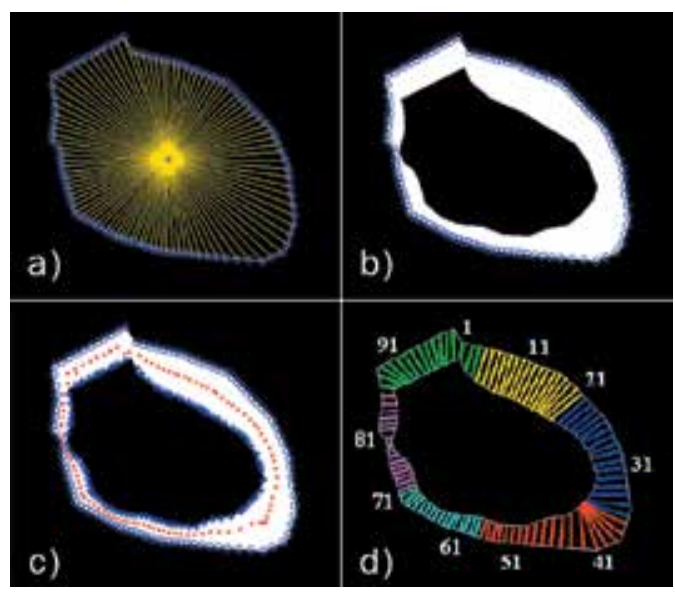

Fuente: autores. 
El corte de dichos radios con los puntos del contorno diastólico es mostrado como círculos de color azul, Fig. 4(b). Seguidamente con base en estos la idea es buscar su correspondencia en el contorno sistólico; para dicho fin, se calculan las componentes normales de cada uno de ellos y se generan perfiles de intensidad hacia el borde del contorno sistólico. Los puntos resultantes son mostrados en la Fig. 4(c) en forma de asteriscos azules. Finalmente, teniendo estos 100 puntos en los contornos diastólico y sistólico final, solo resta calcular el punto medio de cada par de coordenadas (punto 1 contorno diastólico, punto 1 del contorno sistólico y así sucesivamente). El resultado es registrado como puntos rojos en la Fig. 4(c).

\subsubsection{Resultado final}

Al tener las coordenadas de los puntos que conforman la línea central, solo resta trazar la trayectoria normal que sigue cada uno de ellos con base en sus vecinos. El resultado final es ilustrado en la Fig. 4(d), y en donde a su vez se demarcan las 5 zonas divisorias del ventrículo izquierdo en el sistema de área, reportado en [6] para la vista oblicua anterior derecha $30^{\circ}$.

Esta división es ilustrada con el uso de diferentes colores para cada zona como sigue: amarillo, zona Anterobasal; azul, zona Anterolateral; rojo, zona Apical; cyan, zona Diafragmática y violeta, zona Posterobasal. Los radios demarcados en color verde son los correspondientes al plano valvular y no son considerados como una zona divisoria. Seguidamente se calculan cada una de las longitudes radiales mostradas en la Fig. 4(d), las cuales son normalizadas (RNi) por el valor del perímetro del contorno diastólico final [17].

Adicionalmente a esta curva del paciente en estudio (a color), se superponen 3 representaciones más; la primera de ellas consiste en una curva de sinergia promedio, calculada con base en un grupo de pacientes normales (sin ningún tipo de lesión coronaria); las dos restantes corresponden al promedio \pm la desviación estándar, Fig. 5.

Fig. 5. CURVA DE SINERGIA VENTRICULAR, EN LA CUAL SE HAN SUPERPUESTO 3 REPRESENTACIONES MÁS, COMO SON: EL PROMEDIO (O MEDIA ARITMÉTICA), OBTENIDO DE UN GRUPO DE PACIENTES SANOS Y LAS DOS RESTANTES REPRESENTATIVAS DE DICHO PROMEDIO \pm LA DESVIACIÓN ESTÁNDAR

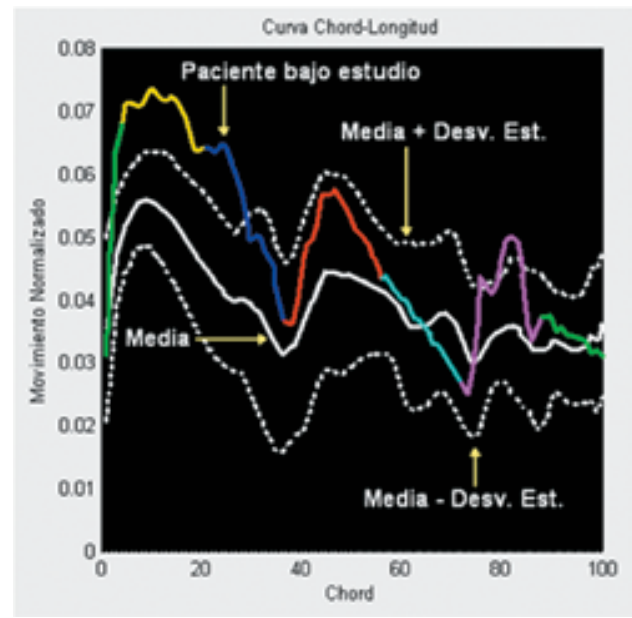

Fuente: autores.

Finalmente, con el propósito de comparar las diferentes regiones del ventrículo, es necesario realizar una estandarización (Zi) dada por (1):

$$
Z_{i}=\left(R N_{i}-M_{i}\right) / D E_{i}
$$

Fundamentada en la media $(M)$ y la desviación estándar $(D E)$ del conjunto de estudios normales, donde $i$ varía de 1 a 100 . Si el valor de $Z i$ es $\geq 2$ se considera que dicho segmento es Hyperkinético; para el caso en que $Z i$ es $\leq-2$ el segmento es ahora Hypokinético y en su defecto si $-2<Z i<2$ este es relacionado como normal [17]. Luego, estos resultados son presentados a nivel porcentual, para cada región ventricular, en función de la cantidad de radios que las componen. 


\section{RESULTADOS}

El sistema desarrollado fue probado utilizando una base de datos obtenida en el IAHULA, compuesta por 19 estudios de ventriculografía. En la Tabla I se relacionan parámetros como el sexo, edad, peso y talla de cada uno de ellos.

$$
\text { TABLA I }
$$

RELACIÓN DE PACIENTES EMPLEADOS EN EL PROCESO CUANTITATIVO

\begin{tabular}{|c|c|c|c|c|}
\hline Paciente & Sexo & Edad & $\begin{array}{c}\text { Peso } \\
\text { (kg) }\end{array}$ & $\begin{array}{l}\text { Talla } \\
(\mathrm{cm})\end{array}$ \\
\hline 1 & Masculino & 44 & 89 & 170 \\
\hline 2 & Masculino & 55 & 61 & 168 \\
\hline 3 & Masculino & 60 & 75 & 163 \\
\hline 4 & Masculino & 42 & 64 & 165 \\
\hline 5 & Masculino & 51 & 52 & 158 \\
\hline 6 & Masculino & 76 & 71 & 168 \\
\hline 7 & Masculino & 52 & 79 & 170 \\
\hline 8 & Masculino & 63 & 65 & 150 \\
\hline 9 & Masculino & 69 & 64 & 165 \\
\hline 10 & Masculino & 70 & 64 & 165 \\
\hline 11 & Masculino & 41 & 65 & 150 \\
\hline 12 & Masculino & 58 & 71 & 168 \\
\hline 13 & Masculino & 49 & 68 & 170 \\
\hline 14 & Masculino & 74 & 88 & 170 \\
\hline 15 & Masculino & 78 & 80 & 170 \\
\hline 16 & Masculino & 63 & 76 & 163 \\
\hline 17 & Masculino & 61 & 54 & 165 \\
\hline 18 & Masculino & 66 & 64 & 165 \\
\hline 19 & Masculino & 75 & 79 & 170 \\
\hline
\end{tabular}

Fuente: autores.

En la Fig. 6, se exponen los resultados obtenidos para un paciente de 69 años de edad, 64 $\mathrm{kg}$ de peso y $165 \mathrm{~cm}$ de talla (paciente \#9). En el inciso (c) es posible notar claramente una disminución generalizada en la contracción sistólica (Hypokinésis), presentándose la mayor reducción en los segmentos Anterobasal (radios amarillos) y Diafragmático (radios cyan), confirmados por la curva de sinergia y cuantificados en el inciso (e) de la figura.

Fig. 6. (A) Y (B) DIÁSTOLE Y SÍSTOLE FINAL, DEMARCANDO SUS CONTORNOS EPICÁRDICOS. (C) APLICACIÓN DEL MÉTODO DE LÍNEA CENTRAL Y DELIMITACIÓN DE LOS 100 RADIOS. (D) CURVA DE SINERGIA VENTRICULAR Y SUPERPOSICIÓN DE LA MEDIA Y LA DESVIACIÓN ESTÁNDAR. (E) ESTIMACIÓN PORCENTUAL DE RADIOS HYPOKINÉTICOS, HYPERKINÉTICOS Y NORMALES EN CADA UNO DE LOS SEGMENTOS VENTRICULARES
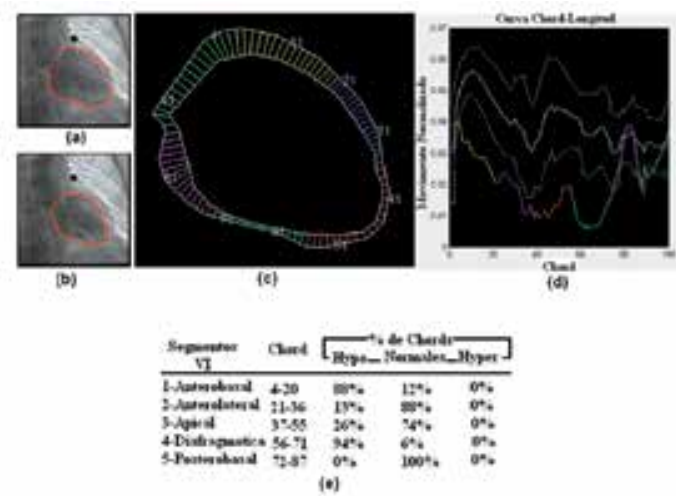

Fuente: autores.

Por su parte la Fig. 7 exhibe la valoración estimada para un paciente de 52 años de edad, 79 $\mathrm{kg}$ de peso y $170 \mathrm{~cm}$ de talla (paciente \#7). En este estudio a simple vista del inciso (c), es difícil determinar si existe o no algún tipo de anormalidad en el movimiento de la pared ventricular; pero gracias a la curva de sinergia es posible detectar un leve aumento de la contracción sistólica (Hyperkinésis) en algunos radios del segmento Anterobasal (amarillos) y posterobasal (violetas), confirmados certeramente por la tabla comparativa del inciso (e).

Fig. 7. (A) Y (B) DIÁSTOLE Y SÍSTOLE FINAL, DEMARCANDO SUS CONTORNOS EPICÁRDICOS. (C) APLICACIÓN DEL MÉTODO DE LÍNEA CENTRAL Y DELIMITACIÓN DE LOS 100 RADIOS. (D) CURVA DE SINERGIA VENTRICULAR Y SUPERPOSICIÓN DE LA MEDIA Y LA DESVIACIÓN ESTÁNDAR. (E) ESTIMACIÓN PORCENTUAL DE RADIOS HYPOKINÉTICOS, HYPERKINÉTICOS Y NORMALES EN CADA UNO DE LOS SEGMENTOS VENTRICULARES.

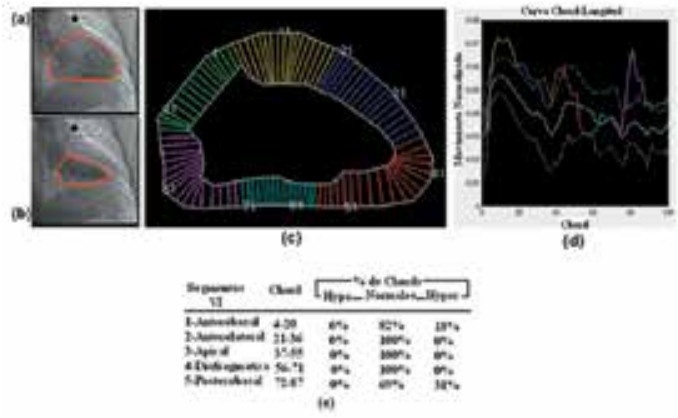

Fuente: autores. 
A nivel cuantitativo se presentan las tablas II y III. La Tabla II, está relacionada con los parámetros obtenidos con la plataforma desarrollada (expuesto a lo largo del artículo) y Tabla III corresponde a la plataforma de referencia empleada en el
IAHULA. En estas se denotan el número de radios obtenidos como hypokinéticos, normales e hyperkinéticos (en función de la media y la desviación estándar), para cada uno de los segmentos en los cuales se divide el ventrículo izquierdo [17].

TABLA II

ANÁLISIS CUANTITATIVO DEL MOVIMIENTO DE LA PARED, EXPRESADA EN FUNCIÓN DE LOS DIFERENTES SEGMENTOS VENTRICULARES. PLATAFORMA DESARROLLADA. D.E. (DESVIACIÓN ESTÁNDAR)

\begin{tabular}{|c|c|c|c|c|c|c|}
\hline \# Radios & Hypo & Normal & Hyper & Hypo & Normal & Hyper \\
\hline Segmento & \multicolumn{3}{|c|}{ Anterobasal } & \multicolumn{3}{|c|}{ Anterolateral } \\
\hline Media & 11,42 & 82,05 & 6,52 & 11,52 & 85,57 & 2,94 \\
\hline D. E. & 23,81 & 24,68 & 14,11 & 27,33 & 28,90 & 12,84 \\
\hline Segmento & \multicolumn{3}{|c|}{ Apical } & \multicolumn{3}{|c|}{ Diafragmático } \\
\hline Media & 21,36 & 77,78 & 0,84 & 12,84 & 85,21 & 1,94 \\
\hline D. E. & 37,56 & 37,15 & 2,71 & 27,15 & 28,67 & 7,16 \\
\hline Segmento & \multicolumn{3}{|c|}{ Posterobasal } & & & \\
\hline Media & 6,94 & 85,26 & 7,78 & & & \\
\hline D. E. & 13,81 & 20,12 & 15,32 & & & \\
\hline
\end{tabular}

Fuente: autores.

TABLA III

ANÁLISIS CUANTITATIVO DEL MOVIMIENTO DE LA PARED, EXPRESADO EN FUNCIÓN DE LOS DIFERENTES SEGMENTOS VENTRICULARES. PLATAFORMA DE REFERENCIA

\begin{tabular}{|c|c|c|c|c|c|c|}
\hline \# Radios & Нypo & Normal & Hyper & Нypo & Normal & Hyper \\
\hline Segmento & \multicolumn{3}{|c|}{ Anterobasal } & \multicolumn{3}{|c|}{ Anterolateral } \\
\hline Media & 12,57 & 86,05 & 1,36 & 8,84 & 86 & 5,15 \\
\hline D. E. & 18,88 & 18,86 & 5,96 & 24,35 & 26,62 & 14,56 \\
\hline Segmento & \multicolumn{3}{|c|}{ Apical } & \multicolumn{3}{|c|}{ Diafragmático } \\
\hline Media & 24,21 & 75,78 & 0,00 & 10,94 & 86,42 & 2,63 \\
\hline D. E. & 38,01 & 38,01 & 0,00 & 28,48 & 28,35 & 5,99 \\
\hline Segmento & \multicolumn{3}{|c|}{ Posterobasal } & & & \\
\hline Media & 6,94 & 92,21 & 0,84 & & & \\
\hline D. E. & 10,71 & 10,76 & 3,67 & & & \\
\hline
\end{tabular}

Fuente: IAHULA.

Es importante resaltar, que por un error porcentual no fue posible extraer a nivel comparativo entre ambos escenarios. La explicación es la siguiente: en la plataforma desarrollada, se buscó una ventana de radios fija, para todos los estudios procesados, como sigue: Zona Anterobasal, radios comprendidos entre el 4-20; Zona Anterolateral, radios comprendidos entre el 21-36; Zona Apical, radios comprendidos entre el 37-55; Zona Diafragmática, radios comprendidos entre el 56-71 y la Zona Posterobasal, radios comprendidos entre el 72-87; como lo demuestran las Fig. 6 y 7; mientras que para la plataforma de referencia, esta distribución de radios por segmentos no es de ninguna manera uniforme, tanto a nivel de número de radios como de inicio y final de los mismos (criterio del especialista).

\section{CONCLUSIONES}

El análisis de anormalidades en el movimiento regional de la pared ventricular, es vital en afectaciones relacionadas a las arterias coronarias. Estudios muestran que dicho movimiento proce- 
de hacia muchos puntos de la cámara ventricular. Basados en este hecho, se implementó un modelo de línea central que cuantifica regionalmente este movimiento a lo largo de 100 radios, clasificándolos en hyperkinéticos, hypokinéticos y normales.

A nivel comparativo con la plataforma de referencia, es de gran relevancia subrayar la variabilidad en los resultados que esta presenta, a nivel del análisis de segmentos hypokinéticos, normales e hyperkinéticos; debido principalmente a la intervención directa del especialista (variadas opiniones, dependiendo del cardiólogo de turno) sobre la selección de los intervalos de procesamiento y análisis. Para finalizar, con el desarrollo de esta plataforma se disminuyó claramente dicho factor en cuestión, con la selección de ventanas fijas (configuradas con la ayuda del especialista) para cada uno de los segmentos analizados.

\section{REFERENCIAS}

[1] J. Mackay and G. Mensah, Atlas of heart disease and stroke, World Health Organization, 2004.

[2] A. Guyton and J. Hall, Textbook of medical physiology, 10th ed., W. B. Saunders, ch. 10. 2001.

[3] S. Fox, Fisiología humana, 10. edición, McGraw-Hill, 2008.

[4] E. Folland, G. Hamilton, S. Larson and J. Kennedy, "The radionuclide ejection fraction: a comparison of three radionuclide techniques whit contrast angiography", Diagnostic Nuclear Medicine, vol. 18, pp.1159-1166, 1977.

[5] N. Ingels, G. Daughters, E. Stinson and E. Alderman, "Evaluation of methods for quantitating left ventricular segmental wall motion in man using myocardial markers as a standard", Circulation, vol. 61, pp. 966-972, 1980.

[6] H. Gelberg, B. Brundage, S. Glantz and W. Parmley, "Quantitative left ventricular wall motion analysis: a comparison of area, chord and radial methods", Circulation, vol. 59, pp. 991-1000, 1979.
[7] R. Leighton, S. Wilt and R. Lewis, "Detection of hypokinesis by a quantitative analysis of left ventricular cineangiograms", Circulation, vol. 50, pp. 121-127, 1974.

[8] S. Antoine, "Extraction et caractérisation du mouvement cardiaque in imagerie scanner multibarrette", Ph.D. dissertation, Université de Rennes, 2006.

[9] A. Bravo, "Simulación y reconstrucción en 4-D del ventrículo izquierdo en imagenología cardiaca". Tesis doctoral, Universidad Simón Bolívar, Venezuela, 2006.

[10] General Electric Company, "Innova 2000, digital cardiovascular x-ray imaging system".

[11] J. Sagardi, "El detector digital en un sistema de imagen cardiovascular", Revista de Física Médica, vol. 3, pp. 35-38, 2002.

[12] F. Sheehan, D. Stewart, H. Dodge, S. Mitren, E. Bolson and B. Brown, "Variability in the measure ment of regional left ventricular wall motion from contrast angiograms, Circulation, vol. 68, pp. 550-559, 1983.

[13] P. Hough, "Method and Means for Recognizing Complex Patterns". U.S. Patent 3069654, 1962.

[14] R. Duda and P. Hart, "Use of the Hough Transformation to Detect Lines and Curves in Pictures", Comm. ACM, vol. 15, pp. 11-15, 1972.

[15] J. Zhang, J. Hu, "Image segmentation based on 2d otsu method with histogram analysis". International Conference on Computer Science and Software Engineering, vol. 6, pp. 105-108, 2008.

[16] J. Canny, "A computational approach to edge detection", IEEE Transactions on Pattern Analysis and Machine Intelligence, vol. 8, pp. 679-698. 1986.

[17] F. Sheehan, E. Bolson, H. Dodge, D. Mathey, J. Schofer, H. Woo. "Advantages and applications of the centerline method for characterizing regional ventricular function". Circul ition, vol. 74, pp. 293-305. [Online]. Available: http://circ.ahajournals. 1986. 